\title{
ESQUEMAS MALADAPTATIVOS TEMPRANOS Y DEPRESIÓN EN PACIENTES JÓVENES HOSPITALIZADOS*
}

\section{Early maladaptive schemas and depression in hospitalized young patients}

\author{
Karla Ivette Meza Arroyo - Centro Educativo (Inicial y Primaria)**, \\ Rocio del Pilar Santayana Rengifo - Servicio Rural y Urbano Marginal \\ de la Salud (SERUMS)***,
}

\begin{abstract}
Resumen
La presente investigación es de tipo cuantitativa con un diseño descriptivo y correlacional, tiene como propósito comparar la relación que existe entre los esquemas maladaptativos tempranos y la depresión en pacientes jóvenes hospitalizados en una Institución de Salud de Lima. La muestra estuvo conformada por 100 pacientes varones con edades oscilantes entre los 18 y 23 años internados en diferentes unidades de hospitalización.

Los resultados arrojaron que el único esquema maladaptativo temprano que se relaciona con la depresión, es el esquema de Negatividad con un $95 \%$ de confianza. Por lo que se concluye que las necesidades no cubiertas en estas personas son la espontaneidad y juego. Además al reprimir sus sentimientos, impulsos y elecciones siendo niños, ocasionaron que en la etapa adulta, vean la vida de forma pesimista.
\end{abstract}

Palabras clave: Esquemas maladaptativos tempranos, depresión, paciente hospitalizado, Esquema maladaptativo de Negatividad.

\begin{abstract}
This research is quantitative, with a descriptive and correlational design. Its objective is to compare the relationship between the early maladaptive schemas and the depression developed in hospitalized young patients from a Healthcare Institution in Lima. The sample consisted of 100 male patients ranging from 18 and 23 years old, admitted to different hospital units. The outcome yielded that the only early maladaptive schema related to depression is the negativity schema with a 95\% confidence interval. Therefore, it is concluded that the unmet needs of these individuals are spontaneity and play as their feelings, impulse and choices were repressed during childhood, thus causing a pessimistic outlook in adulthood.
\end{abstract}

Keywords: early maladaptive schemas, depression, hospitalized, patient

\footnotetext{
* Artículo basado en la tesis de Licenciatura sustentada por las autoras

** Psicóloga. karla.mezaa.21@gmail.com, https://orcid.org/0000-0002-8479-0059

^** Psicóloga.rociosr1692@gmail.com, https://ORCID.org/0000-0003-1890-385X
} 


\section{INTRODUCCIÓN}

El papel del psicólogo ha tomado mayor protagonismo en la actualidad, derribando muchos mitos que opacaban nuestra labor en el bienestar emocional. Sin embargo, aunque existan grupos que por falta de información y/o desconocimiento se resistan a buscar o recibir apoyo de un profesional especializado frente a situaciones adversas, existe otro grupo de personas que lo hacen con mayor y mejor disposición.

En el ámbito hospitalario, encontramos un sector vulnerable, tanto en la salud física como en lo emocional puesto que, la espera de un diagnóstico, el tiempo de hospitalización e información respecto a ello, movilizan sus esquemas y acrecientan la posibilidad de desarrollar trastornos del humor afectando no sólo al paciente, sino también a su grupo primario de apoyo.

Por tanto, nuestra investigación se centró en el estudio de la población hospitalizada en una institución de salud de Lima, conformada por varones jóvenes, con el fin de dar a conocer los esquemas maladaptativos tempranos desarrollados a lo largo de su vida y la relación que existe entre éstos y la depresión (la cual pudo ser desarrollada durante su estancia hospitalaria).

\section{MARCO TEÓRICO}

\section{Esquemas Maladaptativos Tempranos:}

Son creencias profundamente enraizadas, patrones cognitivos y emocionales perjudiciales aprendidos en los primeros años de vida; que aparecen al comienzo de nuestro desarrollo y se repiten a lo largo de nuestra existencia. (Young et al., 2013).

Proporcionan un sentido de predicción y seguridad, son cómodos y familiares, lo que genera resistencia a desprenderse de ellos haciendo difícil su modificación, pues aceptar el daño que causan significaría abandonar la seguridad de saber quiénes somos y cómo es el mundo que nos rodea. Por ello, al estar presentes en momentos significativos, se rehúsan a desaparecer cuando somos adultos, luchando por repetirse cada vez que sea necesario. (Young, Klosko, 2001).
Para Young et al. (2013), estos esquemas son consecuencia de la insatisfacción de las necesidades emocionales nucleares en la infancia, sumado a las experiencias tempranas y temperamento innato del niño, por tal motivo postula lo siguiente:

- Las necesidades emocionales nucleares como el vínculo seguro, autonomía, libertad en la expresión de emociones y necesidades, espontaneidad y juego, y el autocontrol están presentes en todas las personas, variando sólo en el grado de intensidad en el que se muestran.

- Las experiencias infantiles tóxicas constituyen principalmente el origen de los esquemas maladaptativos.

- El temperamento emocional es quien determina la construcción emocional y la manera de reaccionar frente a diversas situaciones, por tal motivo al encontrarse con experiencias infantiles dolorosas y generar una interacción, permiten la formación de esquemas. (p.39).

\section{Dimensiones y principales Esquemas Maladaptativos Tempranos}

Según Rodríguez (2009) "Young identifica dieciocho esquemas maladaptativos tempranos que se agrupan en cinco dimensiones". (p.63)

Estas son:

Dimensión I: Pertenecen a ese grupo las personas que recibieron poca seguridad y estabilidad emocional en la infancia. Dentro de esta dimensión se encuentran:

- Abandono: Creen que las personas significativas en sus vidas los abandonarán.

- Desconfianza: Muestran cautela ante las intenciones de los demás, pues creen que pretenden dañarlos de alguna forma.

- Privación emocional: Creen que sus conexiones emocionales de protección, empatía y cuidados no serán satisfechas.

- Imperfección: Enfatizan el sentimiento de inferioridad e imperfección, por tal motivo creen ser poco merecedores de afecto si se muestran tal cual son.

- Aislamiento social: Creen que no encajarán en la sociedad, motivo por el cual se aíslan al considerarse diferentes a los demás. 
Dimensión II: Se encuentran en este grupo aquellas personas que presentan poca autonomía e independencia, pues fueron llevados a extremos opuestos (sobreprotección/falta de cuidados). Se encuentran:

- Dependencia: Creen necesitar depender de los demás por sentirse incapaces para controlar y manejar sus responsabilidades.

- Vulnerabilidad: Perciben el mundo como un lugar peligroso del cual deben protegerse, por tanto, viven con miedos exagerados por sentirse incapaces de afrontar las adversidades.

- Apego confuso: Perjudican su identidad y desarrollo social al vivir a expensas de personas significativas.

- Fracaso: Sentimiento de inferioridad y creencia exagerada de fracaso inevitable al desarrollar actividades de logro.

Dimensión III: Personas que carecen de límites internos y por ende no respetan las normas de la sociedad, siendo consecuencia de una crianza permisiva en exceso:

- Grandiosidad: Creen ser superiores a los demás confiando en su grandeza y poder. No respetan normas.

- Insuficiente autocontrol: Actúan de manera impulsiva sin medir consecuencias. Poco autocontrol y tolerancia a la frustración

Dimensión IV: Personas que sobreponen la necesidad de los demás por encima de la propia, buscando aprobación para sentirse valorados y evitar consecuencias desagradables.

- Subyugación: Se someten al control de los demás para evitar represalias o abandonos al creer que sus sentimientos y emociones no son importantes como las del resto.

- Autosacrificio: Para no sentirse culpables, anteponen las necesidades de los demás por encima de las propias.

- Búsqueda de aprobación: Buscan la aprobación de los demás, dejando que ellos influyan en su autoestima, toma de decisiones, sentimientos, entre otros.
Dimensión V: Personas que reprimen su espontaneidad para seguir normas rígidas autoimpuestas. Ven el mundo de manera pesimista, mayormente provienen de familias estrictas:

- Negatividad: Perciben su entorno de forma negativa, quejándose y preocupándose constantemente, teniendo la creencia que en cualquier momento cometerán algún error y saldrán perjudicados.

- Inhibición emocional: Se muestran distantes y fríos por miedo a la crítica, inhibiendo su espontaneidad.

- Metas inalcanzables: Presentan estándares internos muy elevados que necesitan satisfacer con perfección, cuidando todos los detalles. Muy críticos consigo mismos y los demás.

- Castigo: Sancionan y se muestran poco tolerantes con personas que no satisfacen sus estándares.

\section{DEPRESIÓN:}

La Organización Mundial de la Salud (s.f.) define la depresión como "un trastorno mental frecuente que se caracteriza por la presencia de tristeza, pérdida de interés o placer, sentimientos de culpa o falta de autoestima, trastornos del sueño o del apetito, sensación de cansancio y falta de concentración".

Por otra parte, Zarragoitía (2011, p. 6) menciona que es "una alteración primaria del estado de ánimo, caracterizada por abatimiento emocional que afecta todos los aspectos de la vida del individuo".

Por lo que se puede inferir que la depresión es un trastorno mental que interfiere en la vida cotidiana de la persona.

\section{Etiología}

Existen diversas causas que intervienen para que una persona padezca depresión. Diversos estudios señalan que las raíces no necesariamente son independientes, pues muchas veces pueden interrelacionarse y deberse a distintos factores, ya sea a nivel genético, biológico (químicos y hormonales), situaciones psicosociales y rasgos de personalidad. 
a) Genético: Según refiere Manios, M. y Rosero, B. (2011) señala que "algunos tipos de depresión tienden a afectar a los miembros de la misma familia, pudiendo heredar una predisposición biológica" (p.49). Es por ello que la depresión no está lejos de ser una enfermedad genética que puede ser transmitida de generación en generación

b) Biológico: Dentro de las causas biológicas influyentes se encuentran los factores químicos y hormonales

- Químicos: Según Manios, M. y Rosero, B. (2011) mencionan que "los neurotransmisores que se alteran son serotonina, acetilcolina, dopamina y las catecolaminas". (p.48)

-Hormonales: Las alteraciones hormonales también contribuyen para desencadenar depresión; tanto en varones como en mujeres, por ello la Hormona Tiroidea, cuando no funciona adecuadamente, puede causar dos tipos de problemas: demasiada hormona tiroidea (hipertiroidismo) y muy poca hormona tiroidea (hipotiroidismo). Cualquiera de estos trastornos puede llevar a la depresión, pero esta tiende a ser más frecuente en el hipotiroidismo. Influyen sobre la forma como se sienten, cómo piensan y cómo se comportan...etc (Avila, 2014, p. 23-24).

c) Psicosocial: Manios, M. y Rosero, B. (2011) mencionan que "la depresión puede ser consecuencia o respuesta a determinadas situaciones traumáticas, como la pérdida de un ser querido, enfermedad, divorcio o situaciones de fuerte estrés" (p.51), por tanto, es importante el análisis del entorno en el que se desenvuelve.

d) Personalidad: Zúñiga et. al. (comp. 2005, p.514) señala que "ciertos tipos de personalidad: oral, dependiente, obsesiva-compulsiva o histérica presentan un mayor riesgo de depresión"

Los individuos con patrones de pensamientos negativos, baja autoestima, sensación de falta de control y tendencia a la preocupación excesiva, son más propensos a padecer depresión. Manios, M. y Rosero, B. (2011).

\section{Planteamiento del problema:}

En la actualidad, se observa que la mayoría de personas optan por quejarse y recriminarse el hecho de repetir situaciones o circunstancias que no aportan nada positivo a sus vidas, generando en sí mismas malestar emocional y constantes fracasos. Esto ocurre desde siempre y es que de manera inconsciente repiten patrones instaurados en su infancia, aprendidos del entorno más cercano, patrones denominados esquemas maladaptativos tempranos, los cuales influyen directamente en su forma de pensar, sentir y actuar, que si bien cuando niños les eran útiles y necesarios, hoy sólo causan molestias, interfiriendo en sus relaciones sociales y proyectos personales, impidiendo también la autorrealización anhelada, llegando en ocasiones al auto sabotaje. Young et al. (2013)

Al ser los esquemas maladaptativos tempranos parten del mundo inconsciente y por ende son difíciles de identificar. Las personas no sólo tienden a echarse la culpa de lo que sucede, sino que también culpan al exterior, sin obtener respuestas ni soluciones, motivo por el cual, en algunos casos, caen en la desolación o en algo más profundo como la depresión. Azabache L. y Rojas B. (2005)

Hoy en día la depresión es considerada una epidemia, ya que es una de las causas de mortalidad más altas a nivel mundial; sin ir demasiado lejos, en nuestro país, se han observado cifras alarmantes de personas que la padecen, muchas de las cuales no tienen claro su concepto ni los riesgos que ocasiona resistirse a una atención adecuada brindada por especialistas, generado en su mayoría por la escasa información, los pocos centros de ayuda en relación a salud mental o por el mismo desinterés que muestran. Esta enfermedad que se instaura de manera silenciosa, afecta el desenvolvimiento general, influyendo directamente en las actividades cotidianas. Ávila (2014).

Con esta investigación, se busca analizar la relación entre los esquemas maladaptativos tempranos y la depresión, en una muestra de varones jóvenes hospitalizados que al encontrarse en circunstancias adversas y con diferentes diagnósticos físicos (los cuales varían en intensidad) los hacen susceptibles a padecer, posteriormente, cambios de humor o activar posibles trastornos del humor como la depresión. 
En base a lo expuesto, el problema queda planteando de la siguiente manera: ¿Cuál es la relación existente entre los esquemas maladaptativos tempranos y la depresión desarrollados en pacientes jóvenes hospitalizados en una Institución de Salud de Lima?

\section{Objetivo General:}

Analizar la relación que existe entre los esquemas maladaptativos tempranos y depresión desarrollados en pacientes jóvenes hospitalizados en una Institución de Salud de Lima.

\section{Objetivos Específicos:}

- Identificar los esquemas maladaptativos tempranos desarrollados en los pacientes jóvenes hospitalizados en una Institución de Salud de Lima.

- Determinar los niveles de depresión presentes en pacientes jóvenes hospitalizados en una Institución de Salud de Lima.

- Analizar si existe relación entre cada uno de los esquemas maladaptativos tempranos y la depresión desarrollados en los pacientes jóvenes hospitalizados en una Institución de Salud de Lima.

\section{MÉTODO:}

\section{Tipo y diseño:}

Investigación de tipo cuantitativo, diseño descriptivo y correlacional.

\section{Participantes:}

La muestra estuvo conformada por 100 pacientes varones con edades oscilantes entre los 18 y 23 años, internados en diferentes unidades de hospitalización en una Institución de Salud de Lima.

\section{Instrumentos:}

Se utilizó el Cuestionario de Esquemas de Young (YSQ-SF 3) validado mediante criterio de jueces en Lima - Perú el año 2013 por Silvia Tamariz Paredes, teniendo como muestra a 64 mujeres adolescentes con edades oscilantes entre 15 y 18 años, con valores iguales o mayores a 0.80 y una confiabilidad con Alfa de Cronbach de 0.935. Consta de 90 preguntas, se aplica de manera individual o grupal.

Asimismo, se utilizó La Escala de Depresión que consta de 20 frases relacionadas a esta problemática, formuladas la mitad en términos positivos y la otra mitad en términos negativos. Tienen gran peso los síntomas somáticos y los cognitivos, con 8 ítems para cada grupo, completándose la escala con dos ítems referentes al estado de ánimo y otros dos a síntomas psicomotores. Confiabilidad con Alfa de Cronbach de 0.79 y 0.92 .

\section{Procedimiento:}

Se pidió por escrito un permiso a la Institución de Salud correspondiente para realizar el trabajo de investigación con la población requerida.

Posteriormente, se realizó un monitoreo para determinar la cantidad de pacientes que cumplían los requisitos y ser parte de la muestra, obteniendo pocos participantes, lo cual obligó a extender el tiempo para la evaluación, realizándolo en dos periodos.

Los instrumentos se administraron de manera colectiva e individual (dependiendo del estado del paciente y de su nivel de comprensión frente a la prueba) usando un tiempo aproximado de 2 horas, siendo supervisado por ambas investigadoras y prestando ayuda cada vez que surgían dudas o interrogantes. Cabe mencionar que se brindó información previa sobre el motivo de la investigación a fin de conocer su disponibilidad y brindar su consentimiento.

Los pacientes se mostraron colaboradores frente a la evaluación.

Finalmente, luego de recabar los datos necesarios, se procedió al análisis cuantitativo en el programa estadístico SPSS para analizar las hipótesis del trabajo respectivo.

\section{RESULTADOS}

A continuación presentaremos los resultados hallados en la muestra de estudio. 
Tabla 1

Frecuencia y porcentaje para los Esquemas maladaptativos tempranos (EMT)

\begin{tabular}{lcccc}
\hline EMT & \multicolumn{2}{c}{ No Instaurado } & \multicolumn{2}{c}{ Instaurado } \\
& $\mathrm{F}$ & $\%$ & $\mathrm{~F}$ & $\%$ \\
\hline Abandono & 63 & $63 \%$ & 37 & $37 \%$ \\
Desconfianza & 55 & $55 \%$ & 45 & $45 \%$ \\
Privación emocional & 63 & $63 \%$ & 37 & $37 \%$ \\
Imperfección & 72 & $72 \%$ & 28 & $28 \%$ \\
Aislamiento & 61 & $61 \%$ & 39 & $39 \%$ \\
Dependencia & 77 & $77 \%$ & 23 & $23 \%$ \\
Vulnerabilidad & 59 & $59 \%$ & 41 & $41 \%$ \\
Apego confuso & 70 & $70 \%$ & 30 & $30 \%$ \\
Fracaso & 76 & $76 \%$ & 24 & $24 \%$ \\
Grandiosidad & 50 & $50 \%$ & 50 & $50 \%$ \\
Insuficiente autocontrol & 61 & $61 \%$ & 39 & $39 \%$ \\
Subyugación & 77 & $77 \%$ & 23 & $23 \%$ \\
Autosacrificio & 38 & $38 \%$ & 62 & $62 \%$ \\
Búsqueda de aprobación & 42 & $42 \%$ & 58 & $58 \%$ \\
Negatividad & 40 & $40 \%$ & 60 & $60 \%$ \\
Inhibición emocional & 44 & $44 \%$ & 56 & $56 \%$ \\
Metas inalcanzables & 16 & $16 \%$ & 84 & $084 \%$ \\
Castigo & 61 & $61 \%$ & 39 & $39 \%$ \\
\hline Nel00 & & & &
\end{tabular}

$\mathrm{N}=100$

En la tabla 1, se observaquelos siguientes esquemas maladaptativos tempranos; Metas inalcanzables (84\%) referido a exigencias internas muy elevadas, Inhibición emocional $(56 \%)$ cohibición de emociones por temor a ser criticados, Negatividad (60\%) percepción del entorno de manera pesimista, Búsqueda de aprobación (58\%) énfasis por obtener el reconocimiento y atención de los demás y Autosacrificio (62\%) interés por satisfacer voluntariamente las necesidades de los demás por encima de las propias; se encuentran presentes en los pacientes jóvenes hospitalizados.

En relación a los niveles de depresión en la muestra estudiada hallamos lo siguiente:
Tabla 2

Frecuencia y porcentaje para los Niveles de Depresión

\begin{tabular}{lcr}
\hline Depresión & $\mathrm{F}$ & $\%$ \\
\hline Dentro de lo normal & 33 & 33,0 \\
Leve & 52 & ${ }^{\circ} 52,0$ \\
Moderada & 11 & 11,0 \\
Intensa & 4 & 4,0 \\
\hline
\end{tabular}

$\mathrm{N}=100$

En la tabla 2, se observa que el $33 \%$ de los pacientes jóvenes hospitalizados en una Institución de Salud de Lima se encuentran dentro de lo normal, el $52 \%$ presenta una depresión leve, el 11\% una depresión moderada y el $4 \%$ un nivel de depresión intensa.

Tabla 3

Correlación de Spearman para el Esquema Negatividad y la Depresión en los pacientes jóvenes hospitalizados

\begin{tabular}{lcl}
\hline & Esquema 15 & Sig. \\
\hline Depresión & $239 *$ &, 017 \\
\hline
\end{tabular}

$* \mathrm{P} \leq .05$

Se encontró una correlación estadísticamente significativa entre el Esquema Negatividad (percepción del entorno de manera pesimista) y la Depresión en los pacientes jóvenes hospitalizados en una Institución de Salud de Lima (r=0.239) al .05. (Tabla 3)

\section{DISCUSIÓN DE RESULTADOS}

La presente investigación tuvo como objetivo encontrar larelaciónentrelosEsquemas Maladaptativos Tempranos y la Depresión desarrollados en pacientes jóvenes de una Institución de Salud de Lima e identificar cuál o cuáles de los 18 esquemas instaurados son los que predominan para dar un posible origen a la depresión.

Al analizar los resultados se encontró que los pacientes jóvenes presentan más de un esquema, los cuales se encuentran instaurados con un porcentaje significativo y se registra que el esquema de Metas 
inalcanzables (84\%) referido a exigencias internas muy elevadas, esquema Inhibición emocional (56\%) cohibición de emociones por temor a ser criticados, esquema Negatividad (60\%) percepción del entorno de manera pesimista, esquema Búsqueda de aprobación (58\%) énfasis por obtener el reconocimiento y atención de los demás y esquema Autosacrificio (62\%) interés por satisfacer voluntariamente las necesidades de los demás por encima de las propias, los que obtuvieron puntajes por encima de 15. Dentro del cual, el esquema Metas inalcanzables fue el que alcanzó mayor puntuación. Éste último hace referencia a la necesidad que tiene la persona por satisfacer sus estándares internos elevados, optando por cumplirlos tajantemente, siendo hipercríticos consigo mismos y con los demás.

Según Young et al. (2013), quienes presentan este esquema necesitan esforzarse de sobremanera para satisfacer sus demandas a fin de evitar la desaprobación y la vergüenza.

Por ello, al contrastar las experiencias recogidas con la literatura, se observa que existe coherencia, pues los pacientes jóvenes de nuestra muestra suelen ser exigentes con su medio y con ellos mismos.

En cuanto a la depresión Beck (1976), menciona que es un desorden del pensamiento, en el que la persona distorsiona la realidad viéndose de forma negativa en la triada cognitiva (el mismo, el mundo y el futuro).

En esta investigación se recogieron niveles porcentuales significativos para su presencia, obteniendo un $52 \%$ de pacientes con Depresión Leve, un $11 \%$ con Depresión Moderada y un $4 \%$ con Depresión Severa en comparación a un 33\% de personas que no la presentaron. Por lo cual, inferimos que un $67 \%$ presenta este diagnóstico en sus diferentes niveles, lo que nos lleva a confirmar el incremento de esta enfermedad en la sociedad y con mayor razón al estar en una situación de hospitalización.

Un punto importante que da sustento a nuestra hipótesis es la correlación significativa que existe entre el esquema de negatividad y la depresión con un $95 \%$ de confianza. Concluyendo por tal, que es el único que guarda relación entre las variables planteadas.
Al mencionar este esquema nos referimos a que la persona percibe su entorno de manera negativa, teniendo la creencia de fracasar en su cotidianidad al cometer errores que lo perjudicarán en el área financiera, laboral o interpersonal, por ende, vive preocupada y quejándose constantemente.

\section{CONCLUSIONES:}

Después de haber contrarrestado información con la literatura relacionada al problema planteado y los resultados obtenidos, en este capítulo se exponen las siguientes conclusiones:

Los esquemas desarrollados en los pacientes jóvenes son: metas inalcanzables $(84 \%)$, inhibición emocional $(56 \%)$, negatividad $(60 \%)$, búsqueda de aprobación (58\%) y autosacrificio (62\%).

Los esquemas desarrollados en los pacientes están situados en dos dimensiones diferentes: el esquema autosacrificio (interés por satisfacer voluntariamente las necesidades de los demás por encima de las propias) y búsqueda de aprobación (énfasis por obtener el reconocimiento y atención de los demás) en la dimensión IV (Hace referencia a las personas que buscan aprobación, puesto que en su infancia no les permitieron vivir sus propios anhelos) y los esquemas negatividad (percepción del entorno de manera pesimista), Inhibición emocional (cohibirse de emociones por temor a ser criticados) y Metas inalcanzables (referido a exigencias internas muy elevadas) en la dimensión $\mathrm{V}$ (Se encuentran aquellas personas que provienen de familias rígidas y que de adultos reprimen sus deseos por seguir normas estrictas a costa de su felicidad).

El esquema de mayor puntuación fue metas inalcanzables con un $84 \%$ (personas que presentan estándares internos elevados), mientras que el de menor puntuación fue el 12 con un $23 \%$ (se someten al control de los demás a fin de evitar represalias o abandono).

En cuanto a la depresión se obtuvo que el $67 \%$ de la población presenta algún nivel de Depresión (leve, moderada o intensa). 
Un $67 \%$ de la muestra estudiada presenta el diagnóstico de depresión en sus diferentes niveles, lo que nos lleva a confirmar el incremento de esta enfermedad en la sociedad y con mayor razón al estar en una situación de hospitalización.

Se halló correlación significativa entre depresión y esquemas maladaptativos sólo en el esquema negatividad (percepción del entorno de manera pesimista) con una confianza del $95 \%$, este a su vez está desarrollado en la dimensión $\mathrm{V}$, que hace referencia a la represión de espontaneidad y placer por seguir normas rígidas a costa de su felicidad.

\section{REFERENCIAS}

Agudelo, D., Lucuní, L. y Santamaría, J. (2008). Evaluación de la depresión en pacientes hospitalizados por distintas enfermedades médicas en la ciudad de Bucaramanga. Pontificia Universidad Bolivariana. BucaramangaColombia. Recuperado de http://www.redalyc. org/html/801/80111670004/

Agudelo, D., Casa, C. Sánchez, D. (2008). Relación entre esquemas maladaptativos tempranos y características de ansiedad y depresión en estudiantes universitarios. Universidad Pontificia Bolivariana, Bucaramanga, Colombia. Recuperado de http://www.scielo.org.co/pdf/rups/ v8n1/v8n1a7.pdf

Ávila, A. (2014). Prevalencia de ansiedad y depresión en adolescentes embarazadas de edades comprendidas de entre 12 y 18 años que se encuentran en riesgo y sin apoyo familiar del proyecto especial Adole- Isis. Universidad Central del Ecuador Facultad de Ciencias Psicológicas. Consultado el 20 de Setiembre del 2016. Recuperado de http://www.dspace.uce.edu. ec/bitstream/25000/3676/1/T-UCE-0007-121.pdf

Azabache L. y Rojas B. (2005). Trampas vitales y locus de control en mujeres víctimas de violencia conyugal en un centro de salud en Lima. (Tesis de Licencitura). Universidad Femenina del Sagrado Corazón. Lima - Perú.

Benites, J., Barcelo, E. y Gelves M. (2016). Características psicológicas de los pacientes con larga estancia hospitalaria y propuesta de protocolo para su manejo clínico. Cir. plást. iberolatinoam, 42 (4). Recuperado de http:// scielo.isciii.es/scielo.php?script=sci_abstract\&pi $\mathrm{d}=$ S0376-7892201600040001

Botto, A., Acuña, J. y Jiménez, J. (2014). La depresión como un diagnóstico complejo. Implicancias para el desarrollo de recomendaciones clínicas. Universidad de Chile. Rev Med Chile 42:

1297-1305. Recuperado de https://scielo.conicyt. $\mathrm{cl} / \mathrm{pdf} / \mathrm{rmc} / \mathrm{v} 142 \mathrm{n} 10 /$ art10.pdf

Carranza, R. (2012). Depresión y características demográficas asociados en estudiantes y líderes universitarios de Lima Metropolitana. Universidad Peruana Unión. Revista Apuntes Universitarios Año II Número 2. Recuperado de https://dialnet. unirioja.es/servlet/articulo?codigo $=4062952$

Chamorro, C. y Poclin, Y. (2016) Esquemas Maladaptativos Tempranos desarrollados en pacientes mastectomizadas pertenecientes al Club de la Mama del Instituto Nacional de Enfermedades Neoplásicas. (Tesis de licenciatura). Universidad Femenina del Sagrado Corazón, Lima, Perú.

Cipriano, D. (2017). Esquemas desadaptativos tempranos y dependencia emocional en estudiantes de 5to año de secundaria de las Instituciones Educativas Públicas de Nuevo Chimbote en el año 2016. Universidad Cesar Vallejo. ChimbotePerú. Recuperado de http://repositorio.ucv. edu.pe/bitstream/handle/UCV/402/cipriano_ dd.pdf?sequence $=1 \&$ is Allowed $=\mathrm{y}$

Ferre F. (2007). Guía de ayuda a pacientes y familiares Afrontar la depresión. Fundación Arpegio-Fundación Lopez-Ibor. Madrid-España. Recuperado de https://consaludmental.org/ publicaciones/Afrontarladepresion.pdf

García, R. (2015). Prevalencia de depresión en pacientes hospitalizados en el servicio de medicina interna en un hospital nacional febrero 2015. Universidad Mayor de San Marcos. Recuperado de http://cybertesis.unmsm.edu.pe/bitstream/ cybertesis/4139/1/Garc\%C3\%ADa_pr.pdf

García, R. (2015). Prevalencia y factores relacionados a depresión en pacientes geriátricos hospitalizados 
en el servicio de medicina interna del Hospital Regional Honorio Delgado Espinoza. Universidad Nacional de San Agustín. Arequipa- Perú. Recuperado de http://repositorio.unsa.edu.pe/ handle/UNSA/298

Hernández, S. (2014). Prevalencia de depresión en pacientes hospitalizados en el servicio de medicina interna. Universidad Nacional de Trujillo. Recuperado de http://dspace.unitru.edu. pe/handle/UNITRU/7929

Manios, M. y Rosero, B. (2011): Manifestaciones y grados de depresión en un grupo de adolescentes de una institución educativa distrital, de la localidad de Suba. Bogotá. Recuperado de https://repository.javeriana.edu.co/bitstream/ handle/10554/9636/Tesis 16-8.pdf;jsessionid=70 ADC715E00069755D9BE3AA6A89DAEA?seq uence $=1$

Organización Mundial de la Salud (2018). Depresión. Recuperado de http://www.who.int/topics/ depression/es/

Pérez, L., Torres, A. (2015). Esquemas Maladaptativos Tempranos en madres adolescentes pertenecientes a un centro de atención residencial (CAR). (Tesis de licenciatura). Universidad Femenina del Sagrado Corazón, Lima, Perú.

Restrepo, A. (2014). Esquemas maladaptativos y estrategias de afrontamiento en sujetos diagnosticados con VIH. Universidad Cooperativa Colombiana, 12(22). Recuperado de http://www. sidastudi.org/resources/inmagic-img/DD27505. pdf

Restrepo,A. Y. y Zambrano Cruz, R. (2013). Esquemas maladaptativos tempranos en pacientes hipertensos controlados en Segovia, Colombia. Pensando Psicología, 9(16), 69-75. Recuperado de https://
www.researchgate.net/profile/Renato_ZambranoCruz/publication/263658511_Esquemas_ maladaptativos_tempranos_en_pacientes_ hipertensos_controlados_en_Segovia_Colombia/ links/00b7d53b8738fbb82d000000/Esquemasmaladaptativos-tempranos-en-pacienteshipertensos-controlados-en-Segovia-Colombia. pdf

Rodríguez, E. (2009). La terapia centrada en esquemas de Jeffrey Young. Avances psicológicos, 17 (1), 59 - 74. Recuperado de http://www.unife.edu.pe/ pub/revpsicologia/edgarrodriguez.pdf

Rodríguez, E. (2014) Clase de psicología cognitivo conductual. Universidad Femenina del Sagrado Corazón. Lima-Perú.

Tamariz, S. (2013). Esquemas Maladaptativos Tempranos en adolescentes diagnosticadas con maltrato de una institución residencial en Lima. (Tesis de licenciatura). Universidad Femenina del Sagrado Corazón, Lima, Perú.

Young, J \& Klesko, J. (2001). Reinventa tu vida. Cómo superar las actitudes negativas y sentirse bien de nuevo. Buenos Aires: Paidós.

Young, J, Klosko, J. \& Weishaar, M. (2013). Terapia de Esquemas: Guía práctica Guide. Bilbao: Desclée de Brouwer.

Zarragoitía (2011). Depresión generalidades y particularidades.La Habana: Ecimed.Recuperado de http://newpsi.bvs-psi.org.br/ebooks2010/pt/ Acervo_files/depresion-cuba.pdf

Zuñiga et. al (2005). Los trastornos del estado de ánimo. Revista Mexicana de Investigación en Psicología 6 (11). Recuperado de http://www. revista.unam.mx/vol.6/num11/art110/nov_art110. pdf

Fecha de recepción: 3 de junio 2019

Fecha de aceptación: 3 de setiembre 2019 